\title{
Utilização da realidade virtual na doença de Alzheimer: uma revisão bibliográfica
}

Use of virtual reality in Alzheimer's

disease: a literature review

FisiSenectus. Unochapecó Ano 7, n. 1 - Jan/Jun. 2019 p. $61-68$

Alice de Leon Figueiredo. alicinha2304@hotmail.com

Acadêmica do Curso de Graduação em Fisioterapia na Universidade Estadual do Centro-Oeste (UNICENTRO).

Tainá Cazuni Meneghetti. taina1909@hotmail.com

Acadêmica do Curso de Graduação em Fisioterapia na Universidade Estadual do Centro-Oeste (UNICENTRO).

Elizama de Gregório. elizamagregorio@outlook.com

Fisioterapeuta. Mestre em Ciências Biológicas-Fisiologia pela Universidade Federal do Rio Grande do Sul (UFRGS).

Ana Carolina Dorigoni Bini. ana.carolina.db@hotmail.com

Fisioterapeuta. Doutoranda no Programa de Ciências Farmacêuticas. Professora do Departamento de Fisioterapia da Universidade Estadual do Centro Oeste (UNICENTRO). Mestre em Biotecnologia Aplicada à Saúde da Criança e do Adolescente pela Faculdades Pequeno Príncipe.

\section{Resumo}

Introdução: a Doença de Alzheimer (DA) é neurodegenerativa e progressiva, ocasionando uma série de mudanças na estrutura cerebral e incapacidades funcionais, dificultando a realização das atividades de vida diárias e sociais, necessitando de alternativas eficientes no manejo da doença. Nesse sentido, a utilização da realidade virtual (RV) vem sendo bastante visada na área da pesquisa, pela possibilidade de o paciente vivenciar experiências semelhantes às diárias, capazes de explorar várias emoções, assim como a cognição e aptidão física, tornando-a uma estratégia interessante na reabilitação funcional física e cognitiva, útil tanto na avaliação quanto no tratamento de diversas patologias. Objetivo: analisar as diversas formas de utilização da RV no tratamento da sintomatologia em indivíduos que possuem DA. Metodologia: trata-se de uma revisão bibliográfica que buscou identificar as diferentes formas de utilização da RV em indivíduos com DA. A busca foi realizada nas seguintes bases de dados eletrônicas: PubMed; PeDro; EBSCO, Lilacs e SciELO. Depois dos critérios de inclusão e exclusão, foram selecionados quatro artigos científicos. Resultados: a literatura apoia o uso da RV como instrumento de tratamento e avaliação de pacientes com DA, tendo resultados satisfatórios como uma técnica eficaz e que facilita a análise de evolução e de estado desses pacientes. Conclusão: a RV tem se mostrado promissora no tratamento e avaliação de pacientes com déficits cognitivos associado à DA. Porém, novos estudos fazem-se necessários para que seja possível eleger os melhores recursos de RV para atender mais adequadamente pacientes com DA e suas necessidades específicas.

\section{Palavras-chave}

Realidade virtual; Doença de Alzheimer; Idoso.

\section{Fisiß̊nectus}




\begin{abstract}
Introduction: Alzheimer's disease (AD) is neurodegenerative and progressive, causing a series of changes in the cerebral structure and functional incapacities, making difficult the accomplishment of daily and social life activities, present and desired throughout society, necessitating efficient alternatives in the management of the disease. In this sense, the use of virtual reality (VR) has been very focused on the research area, for the possibility of experiencing similar experiences to the daily ones, capable of exploring various emotions, as well as cognition and physical fitness, making virtual reality an interesting strategy in physical and cognitive functional rehabilitation, useful both in the evaluation and treatment of several pathologies. Objective: to analyze the various ways of using VR to treat symptomatology in individuals with AD. Methodology: This study is a literature review that sought to identify the different ways of using VR in individuals with AD. The search was performed in the following electronic databases: PubMed; PeDro; EBSCO, Lilacs and SciELO. After the inclusion and exclusion criteria, four scientific articles were selected. Results: The literature supports the use of VR as an instrument for the treatment and evaluation of patients with AD, with satisfactory and encouraging results being an effective technique that facilitates the analysis of the evolution and status of these patients. Conclusion: We conclude that virtual reality has shown promise in the treatment and evaluation of patients with cognitive deficits associated with Alzheimer's disease. However, new studies are needed so that we can choose the best RV resources to better serve patients with AD and their specific needs.
\end{abstract}

\title{
Keywords
}

Virtual Reality; Alzheimer's disease; Aged.

\section{Introdução}

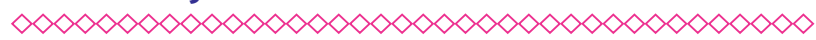

A Doença de Alzheimer (DA) é neurodegenerativa caracterizada por uma série de mudanças na estrutura do cérebro, conexões perdidas, inflamação e eventual morte de células cerebrais. Tais mudanças levam à perda de memória, mudanças no pensamento e de outras funções cerebrais ${ }^{1}$ que geralmente são diagnosticados com o enveIhecimento, processo natural que ocorre gradativamente ocasionando uma série de alterações no organismo, provocando perdas progressivas nas reservas fisiológicas e predispondo o indivíduo a um maior risco de desenvolver múltiplas patologias, que, em diferentes âmbitos, podem alterar sua qualidade de vida $(\mathrm{QV})^{2}$.

Segundo estimativa realizada nos Estados Unidos, em 2050, os casos de DA duplicarão, chegando a uma quantidade aproximada de 13,8 milhões de idosos com a demência ${ }^{3}$. A sua prevalência é associada à idade e de característica crescente, sendo 9\% para indivíduos com idade acima de 65 anos, para indivíduos com mais de 85 anos, 34\% e $43 \%$ para idosos com idade acima de 95 anos $^{4}$.
As alterações advindas do envelhecimento atingem todos os idosos de forma gradual e cumulativa gerando incapacidades funcionais, ocasionando dificuldades na realização das atividades de vida diárias e sociais, presentes e desejadas por toda a sociedade 5 .

Atualmente, as alternativas de tratamentos psicológicos avançaram significativamente, com a criação de vários protocolos explorando o mundo virtual. Essas iniciativas têm se apresentado eficientes no tratamento, inclusive de demências, sendo importante destacar o significativo papel das tecnologias nesse contexto ${ }^{6}$.

A utilização da realidade virtual (RV) vem sendo bastante visada em pesquisas, pela possibilidade de proporcionar ao paciente a vivência de experiências semelhantes ao cotidiano, capazes de explorar várias emoções, assim como a cognição e aptidão física, tornando a RV uma estratégia interessante na reabilitação funcional física e cognitiva, útil tanto na avaliação quanto tratamento de diversas patologias ${ }^{6}$.

A RV possibilita criar ambientes e situações difíceis de serem vivenciadas na vida diária, 
principalmente por se tratar de idosos demenciados, por esse motivo, apresenta-se como uma ferramenta positiva na reabilitação de alguns quadros, bem como na manutenção da qualidade de vida dos idosos com DA, trazendo treinamentos estimulantes e diferenciados dos tratamentos habituais aos quais esses pacientes estão habituados ${ }^{7}$.

Segundo achados da literatura, a RV é utilizada para avaliar características específicas que indicam disfunções cognitivas e deficiências intelectuais, assim como déficits em pacientes com Parkinson, criando situações cotidianas como fazer compras, percorrer o caminho até o supermercado, cozinhar, incluindo funções cognitivas como hesitações e tomadas de decisões. Há uma grande variedade de aplicações da RV na área da saúde, abrangendo todas as fases, desde a avaliação até a reabilitação, em diversas patologias, incluindo as demências ${ }^{8}$. 0 presente artigo de revisão da literatura buscou analisar as diversas formas de utilização da RV no tratamento da sintomatologia em indivíduos que possuem DA.

\section{Metodologia}

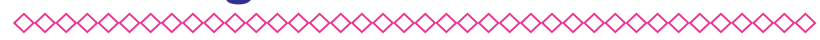

0 presente estudo é uma revisão da literatura que objetivou identificar as diferentes formas de utilização da RV em indivíduos com DA. A busca por literatura foi realizada nas seguintes bases de dados eletrônicas: PubMed; PeDro; EBSCO, Lilacs e SciELO, no mês de outubro do ano de 2018. Devido à ampla quantidade de nomenclaturas para doença de Alzheimer e para realidade virtual, foi selecionado somente "Alzheimer's disease" e "virtual reality" como palavras-chave.

\section{Critérios de elegibilidade dos estudos}

Os estudos selecionados para análise foram os que apresentavam as duas palavras-chave no seu título, os estudos randomizados controlados, somente os com pessoas que possuam DA, descartando outros tipos de demência, e artigos que tenham acesso online livre na integra. Não foi imposto limite temporal para os artigos.

\section{Seleção e extração de dados dos artigos}

A seleção dos artigos foi realizada por três leitores separadamente. A análise iniciou-se pela leitura do título dos textos nas bases de dados e então os que possuíssem as duas palavras-chave no título passavam para o exame dos resumos. A seguir, os artigos selecionados passaram pela leitura do texto na íntegra.

Os dados sobre os participantes e intervenções, os resultados significativos encontrados e a conclusão extraídos dos artigos selecionados foram 0 objetivo do estudo. 0 fluxograma com as etapas de seleção está exposto na Figura 1, e apresenta as fases utilizadas para a seleção dos artigos.

A pesquisa inicial com as palavras-chave nas bases de dados citadas resultou em um total de 77 estudos. Durante a leitura dos títulos foram excluídos 64 artigos por não aderirem ao objetivo desta revisão ou por não conterem as duas palavras-chave no título. Depois da leitura dos resumos, foram excluídos nove artigos, pois suas amostras não eram somente com indivíduos que possuam DA. Ao final, restaram somente quatro artigos para leitura na integra e análise da metodologia e resultados.

\section{Resultados}

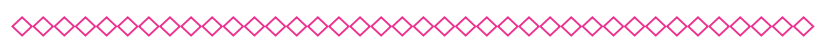

Os resultados da análise dos estudos estão dispostos no Quadro 1, o qual mostra os autores e o ano do estudo, os objetivos, os participantes e os procedimentos, os resultados significativos e a conclusão. Nota-se que a utilização da RV pode se dar de diferentes maneiras e configurações e para diferentes objetivos.

\section{Discussão}

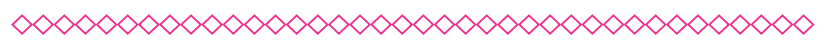

O presente estudo buscou investigar por meio de uma revisão da literatura a aplicabilidade da RV em suas diferentes aplicações em indivíduos com DA. A utilização da RV se deu como instrumento avaliativo para ajustes posturais compensatórios, 
habilidades de compreensão de diferentes representações espaciais, e como teste de navegação virtual, foi também empregado como treinamento de sincronização de dados mentais para memória espacial.

Em um estudo realizado por Manera ${ }^{7}$, utilizando a RV para treinamento de memória em comparação com o treinamento clássico em papel, os participantes relataram preferir a atividade utilizando a RV decorrente de ser uma experiência envolvente, imersiva e motivadora, mostrando algumas das vantagens da utilização desse instrumento ${ }^{7}$.

Os indivíduos com DA e demais demências apresentam sérias alterações cognitivas, como perda de memória e demais alterações no físico e nas habilidades mentais. Davis ${ }^{8}$ relata que um dos sintomas mais perigosos é a perda da capacidade de orientação, esses pacientes possuem um alto risco de se perder. Em estágios iniciais da doença, cerca de $55 \%$ das pessoas têm a capacidade de orientação alteradas ${ }^{8}$.

0 estudo de Serino ${ }^{9}$, observando esse déficit, usou a RV como um instrumento para treinar a capacidade de orientação e memória espacial em indivíduos saudáveis e em pessoas com DA, e teve como resultado a melhora significativa na memória espacial de ambos os grupos, mostrando a eficácia desse recurso ${ }^{9}$.

Davis ${ }^{8}$ utilizou o Virtual Senior Living, que é um programa em RV que busca estimular a orientação pela imitação de atividades da realidade, e teve resultados promissores na utilização de sugestões ambientais com cores e formas diferentes para facilitar a orientação dos pacientes e para memorizar e reconhecer os cômodos em relação a ambientes virtuais que não possuíam essas sugestões visuais ${ }^{8}$.

0 estímulo e o exercício cognitivo trazem diversos benefícios, entre eles, a estimulação de mudanças neuroplásticas. 0 treinamento utilizando a RV oportuniza maior envolvimento e eficácia, ajudando e beneficiando as atividades de vida diária $^{8,10}$. Macedo ${ }^{11}$ mostrou por meio de revisão sistemática que a RV sendo usada para o treinamento cognitivo em indivíduos com comprometimento leve e com demência apresenta resultados positivos na melhora da atenção, memória e execução"11.
Serino ${ }^{12}$, em seu estudo, utilizou a RV para teste cognitivo, que era composto de um mapa virtual no qual o indivíduo deveria memorizar a localização do objeto na primeira etapa e, na segunda, o mesmo mapa, porém vazio, era utilizado para o paciente recuperar a posição do objeto. 0 estudo apresentou como resultado que os indivíduos com comprometimento cognitivo leve amnésico tiveram scores significativamente inferiores na primeira fase, estando relacionado com a dificuldade para codificar e armazenar uma representação espacial. Enquanto os pacientes com DA tiveram resultados significativamente inferiores ao grupo controle na segunda tarefa ${ }^{12}$.

Sendo assim, a literatura apoia uso da RV como instrumento de tratamento e avaliação de pacientes com DA, tendo resultados satisfatórios e animadores, considerando-se uma técnica eficaz e que facilita a análise de evolução e de estado dos pacientes com $\mathrm{DA}^{13}$.

\section{Conclução}

$\infty \infty \infty \times \infty \times \infty \times \infty \times \infty \times \infty \times \infty \times \infty \times \infty \times \infty \times \infty)$

Conclui-se que a RV tem se mostrado promissora no tratamento e avaliação de pacientes com déficits cognitivos associado à DA. Porém, novos estudos são necessários para que se possa eleger os recursos de RV mais promissores para melhor atender pacientes com DA e suas necessidades específicas.

\section{Referências}

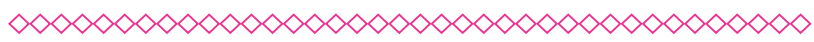

1. Storti LB, Quintino DT, Silva NM, Kusumota L, Marques S. Neuropsychiatric symptoms of the elderly with Alzheimer's disease and the family caregivers' distress. Rev Latino-Am. Enfermagem [Internet]. 2016; 24:e2751.

2. Kim S. Cognitive rehabilitation for elderly people with early-stage Alzheimer's disease. J of Physical Therapy Science. 2015;27(2):543-6.

3. Moon H, Dilworth-Anderson P. Baby boomer caregiver and dementia caregiving: Findings from 
the national study of caregiving. Age and Ageing 2015;44(2):300-6.

4. Ferreti F, Gauer APM, Tomazel EF, Sá CA, Corraldo VS. Capacidade funcional do idoso com dignóstico indicativo de Alzheimer. FisiSenectus Unochapecó. 2014;2(1):33-41.

5. Tomomitsu MRSV, Perracini MR, Neri A L. Fatores associados à satisfação com a vida em idosos cuidadores e não cuidadores. Ciência \& Saúde Coletiva. 2014;19(8):3429-3440.

6. Díaz-Perez E, Flórez-Lozano JA. Virtual reality and dementia. Rev de Neurologia. 2018;66(10):344-52.

7. Manera V, Chapoulie E, Bourgeois J, Guerchouche R, David R, Ondre JJ, et. al. A feasibility study with image-based rendered virtual reality in patients with mild cognitive impairment and dementia. PLoS One. 2016;11(3):e0151487

8. Davis R, Ohman J. Wayfinding in ageing ande Alzheimer's disease within a virtual sênior residence: study protocol. Journal of Advanced Nursing. 2016;72(7):1677-88.

9. Serino S, Pedroli E, Tuena C, De Leo G, StrambaBadiale M, Goulene K, et al. A novel virtual reality- based training protocol for the enhancement of the "mental frame syncing" in individuals with Alzheimer's disease: a development-ofconcept trial. Frontiers in Aging Neurocience. 2017;(9):240.

10. Doninger GM, Beeri MS, Bahar-Fuchs $A$, Gottlier A, Tkchov A, Kenan H, Livny A., et.al. Virtual reality-based cognitive-motor training for middle-aged adults at high Alzheimer's diseaserisk: A randomized controlled trial. Alzheimer Dementia (N Y). 2018(4):118-29

11. Macedo M, Marques A, Queiros C. Realidade virtual na avaliação e no tratamento da esquizofrenia: uma revisão sistemática. Jornal brasileiro de psiquiatria. 2015; 64;1.

12. Serino S, Morganti F, Di Stefano F, Riva G. Detenting early egocentric and allocentric impairments deficits in Alzheimer's disease: an experimental study with virtual reality. Frontiers in Aging Neurocience. 2015;7:88.

13. Tarnanas I, Schlle W, Tsolaki M, Muri R, Mosimann U, Nef T. Ecological Validity of Virtual Reality Daily Living Activities Screening for Early Dementia: Longitudinal Study. JMIR Serious Games. 2013;1(1):e1. 


\section{Anexos}

Figura 1 - Fluxograma com o processo de seleção dos estudos

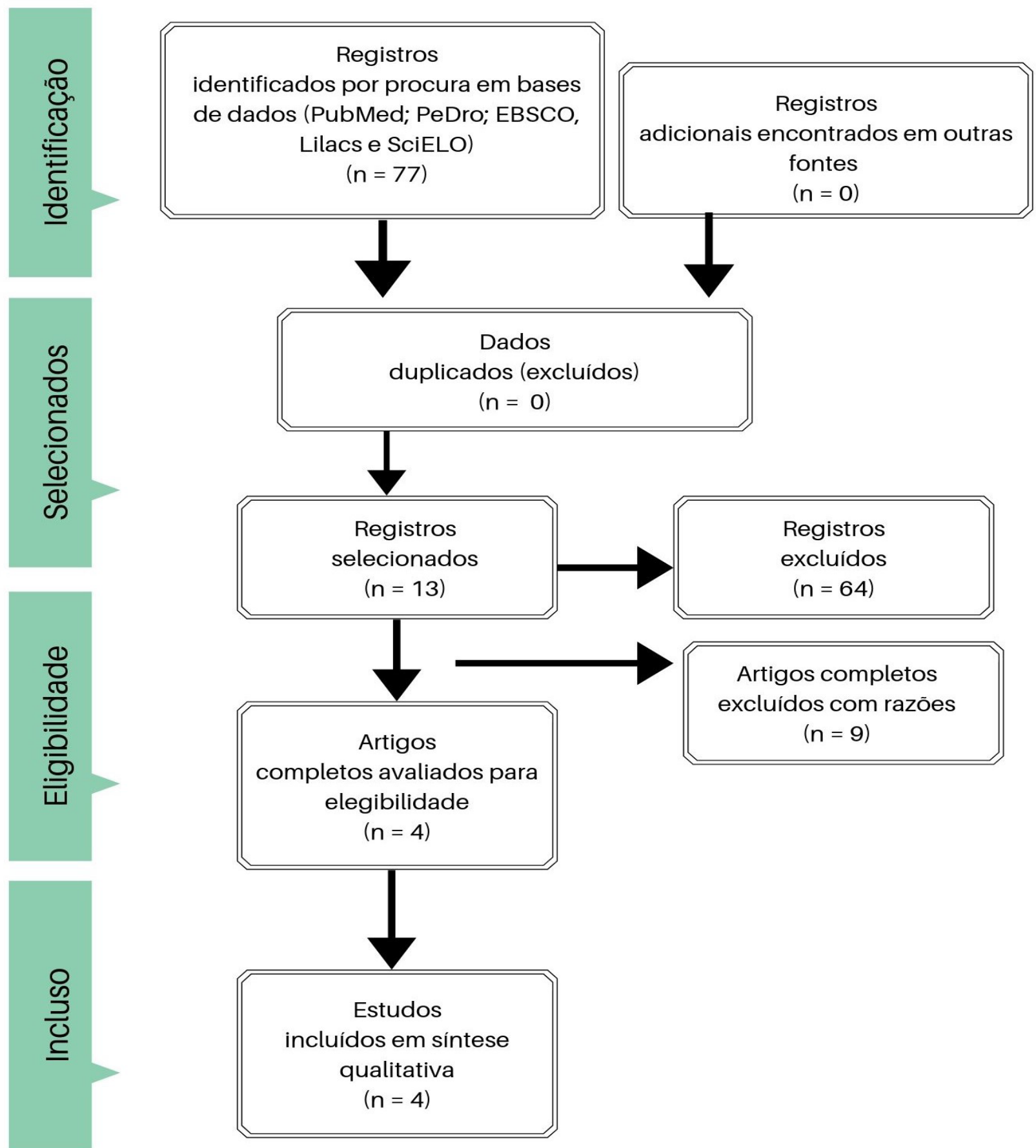

Fonte: Elaborada pelas autoras 
Quadro 1 - Quadro com os dados analisados

\begin{tabular}{|c|c|c|c|c|}
\hline $\begin{array}{c}\text { Autor e } \\
\text { ano de } \\
\text { publicação }\end{array}$ & Objetivo & Métodos & Resultados & Conclusões \\
\hline $\begin{array}{l}\text { SERINO et al } \\
(2017)\end{array}$ & $\begin{array}{c}\text { Eficácia de um } \\
\text { novo protocolo } \\
\text { de treinamento } \\
\text { baseado em RV } \\
\text { focado para a } \\
\text { "sincronização de } \\
\text { quadros mentais" } \\
\text { em diferentes } \\
\text { estados espaciais } \\
\text { em indivíduos com } \\
\text { DA. }\end{array}$ & $\begin{array}{c}28 \text { participantes } \\
\text { (Controle n=10; } \\
\text { DA n=10; Saudável } \\
\text { RV n=8) } \\
\text { Tratamento RV: } \\
10 \text { sessões, três } \\
\text { fases por sessão: } \\
\text { treinamento, } \\
\text { "codificação" e fase } \\
\text { de "recuperação". } \\
\text { Controle com } \\
\text { tratamento } \\
\text { cognitivo } \\
\text { tradicional. }\end{array}$ & $\begin{array}{l}\text { Houve uma melhora } \\
\text { significativa na } \\
\text { memória espacial } \\
\text { de longo prazo após } \\
\text { o treino em RV no } \\
\text { grupo DA e indivíduos } \\
\text { saudáveis. }\end{array}$ & $\begin{array}{c}\text { Em suma, a } \\
\text { RV pode ser } \\
\text { considerada como } \\
\text { uma ferramenta } \\
\text { incorporada } \\
\text { avançada adequada } \\
\text { para tratar } \\
\text { deficiências de } \\
\text { memória espacial. }\end{array}$ \\
\hline $\begin{array}{c}\text { GAGO et al. } \\
(2016)\end{array}$ & $\begin{array}{c}\text { Estudar os } \\
\text { ajustes posturais } \\
\text { compensatórios } \\
\text { provocados (CPAs) } \\
\text { em um paradigma } \\
\text { sensorial } \\
\text { conflitante com } \\
\text { deslocamentos } \\
\text { visuais } \\
\text { imprevisíveis } \\
\text { usando óculos de } \\
\text { realidade virtual. }\end{array}$ & $\begin{array}{c}40 \text { participantes } \\
\text { (19 controles, } 21 \\
\text { com DA, divididos } \\
\text { em relação } \\
\text { ao equilíbrio). } \\
\text { Tratamento de RV } \\
\text { usando análises } \\
\text { cinemáticas } \\
\text { de frequência- } \\
\text { tempo em baixa } \\
\text { frequência (LB; } 0,3- \\
\text { 1,5 Hz; estratégia } \\
\text { mecânica) e alta } \\
\text { frequência (HB; } 1,5- \\
\text { 3,5 Hz; estratégia } \\
\text { cognitiva). }\end{array}$ & $\begin{array}{c}\text { O grupo AD } \\
\text { "caidores" } \\
\text { apresentou CPAs LB } \\
\text { de maior frequência, } \\
\text { refletindo sua } \\
\text { pior estabilidade } \\
\text { postural inerente. Os } \\
\text { pacientes com DA } \\
\text { apresentaram um } \\
\text { atraso na reação de } \\
\text { PCR-A. }\end{array}$ & $\begin{array}{l}\text { A reação mais lenta } \\
\text { por CPA na DA pode } \\
\text { ser um reflexo de } \\
\text { diferentes recursos } \\
\text { cognitivos, } \\
\text { incluindo } \\
\text { autopercepção do } \\
\text { esquema corporal, } \\
\text { movimento visual, } \\
\text { percepção de } \\
\text { profundidade } \\
\text { ou um estado } \\
\text { diferente de medo } \\
\text { e / ou ansiedade. }\end{array}$ \\
\hline
\end{tabular}

(Continua) 


\begin{tabular}{|c|c|c|c|c|}
\hline $\begin{array}{c}\text { Autor e } \\
\text { ano de } \\
\text { publicação }\end{array}$ & Objetivo & Métodos & Resultados & Conclusões \\
\hline $\begin{array}{l}\text { SERINO et } \\
\text { al. (2015) }\end{array}$ & $\begin{array}{c}\text { Comparar o } \\
\text { desempenho de } \\
\text { indivíduos com } \\
\text { comprometimento } \\
\text { cognitivo leve } \\
\text { amnéstico } \\
\text { (AMCI), com DA } \\
\text { e grupo controle } \\
\text { (GC) usando um } \\
\text { procedimento } \\
\text { baseado VR } \\
\text { que avaliar as } \\
\text { habilidades } \\
\text { em codificar, } \\
\text { armazenar e } \\
\text { sincronizar } \\
\text { diferentes } \\
\text { representações } \\
\text { espaciais. }\end{array}$ & $\begin{array}{c}45 \text { participantes } \\
\text { (AD=15, AMCI =15, } \\
\text { GR=15). } 2 \text { tarefas, } \\
\text { na } 1 \text { indicaram } \\
\text { em um mapa } \\
\text { real a posição do } \\
\text { objeto que haviam } \\
\text { memorizado, } \\
\text { na } 2 \text { tarefa eles } \\
\text { recuperaram sua } \\
\text { posição de uma } \\
\text { versão vazia da } \\
\text { mesma sala virtual, } \\
\text { começando de uma } \\
\text { posição diferente. } \\
\text { Tiveram } 3 \text { tentativas } \\
\text { diferentes, } \\
\text { dependendo da } \\
\text { localização do } \\
\text { objeto na fase de } \\
\text { codificação. }\end{array}$ & $\begin{array}{l}\text { Os pacientes com } \\
\text { AMCI apresentaram } \\
\text { um desempenho } \\
\text { significativamente } \\
\text { pior na terceira } \\
\text { tentativa da primeira } \\
\text { tarefa, demonstrando } \\
\text { um déficit na } \\
\text { capacidade de } \\
\text { codificar e armazenar } \\
\text { uma representação } \\
\text { independente do } \\
\text { ponto de vista } \\
\text { alocêntrico. Os } \\
\text { pacientes com } \\
\text { DA apresentaram } \\
\text { desempenho } \\
\text { significativamente } \\
\text { pior quando } \\
\text { comparados ao GC na } \\
\text { segunda tarefa. }\end{array}$ & $\begin{array}{c}\text { Esses achados } \\
\text { fornecem uma } \\
\text { visão inicial } \\
\text { sobre as bases } \\
\text { cognitivas do } \\
\text { comprometimento } \\
\text { clínico em } \\
\text { pacientes com ICM } \\
\text { e DA. }\end{array}$ \\
\hline $\begin{array}{l}\text { CUSHMAN, } \\
\text { STEIN, } \\
\text { DUFFY } \\
(2008)\end{array}$ & $\begin{array}{c}\text { Comparar um teste } \\
\text { de navegação real } \\
\text { com uma versão de } \\
\text { realidade virtual } \\
\text { (VR) que simula o } \\
\text { mesmo ambiente } \\
\text { de navegação. }\end{array}$ & $\begin{array}{l}87 \text { participantes } \\
\text { (35 controles } \\
\text { jovens (YNCs), } 26 \\
\text { controles adultos } \\
\text { (ONCs), } 12 \text { com } \\
\text { comprometimento } \\
\text { cognitivo leve (MCI), } \\
14 \text { com DA precoce } \\
\text { (EAD). Os testes } \\
\text { de navegação do } \\
\text { mundo real e do } \\
\text { ambiente virtual } \\
\text { consistiam em } \\
\text { oito subtestes } \\
\text { correspondentes, e } \\
\text { foram concluídas em } \\
3 \text { dias. }\end{array}$ & $\begin{array}{l}\text { Teve correlações } \\
\text { entre os déficits } \\
\text { de navegação } \\
\text { reais e virtuais que } \\
\text { aumentaram entre os } \\
\text { grupos de YNC a ONC, } \\
\text { MCI e EAD. Análises } \\
\text { do desempenho do } \\
\text { subteste mostraram } \\
\text { perfis semelhantes } \\
\text { de prejuízo nos testes } \\
\text { reais e virtuais em } \\
\text { todos os quatro } \\
\text { grupos de assuntos. } \\
\text { Todos os sujeitos } \\
\text { do ONC, MCI e EAD } \\
\text { tiveram maior } \\
\text { dificuldade no testes } \\
\text { de auto-orientação e } \\
\text { localização de cena. } \\
\text { Pacientes com MCI e } \\
\text { EAD também tiveram } \\
\text { prejuízo na lembrança } \\
\text { verbal sobre ambos } \\
\text { os ambientes de } \\
\text { teste. }\end{array}$ & $\begin{array}{l}\text { Concluiu-se que } \\
\text { os testes de } \\
\text { navegação virtual } \\
\text { revelam déficits no } \\
\text { envelhecimento } \\
\text { e DA associados } \\
\text { a riscos } \\
\text { potencialmente } \\
\text { graves para os } \\
\text { pacientes e para a } \\
\text { comunidade. }\end{array}$ \\
\hline
\end{tabular}

